\title{
Anxiety at First and Subsequent Pregnancies and Its Associated Factors: A Historical Cohort Study From Northeastern Thailand
}

\author{
Rukmanee Butchon, M.Sc. ${ }^{1}$, Tippawan Liabsuetrakul, M.D., Ph.D. ${ }^{2}$, \\ Theparat Bumpenboon, M.D. ${ }^{3}$, Yot Teerawattananon, M.D., Ph.D. ${ }^{1}$ \\ ${ }^{1}$ Health Intervention and Technology Assessment Program, Mueang, Nonthaburi 11000, Thailand. \\ ${ }^{2}$ Epidemiology Unit, Faculty of Medicine, Prince of Songkla University, Hat Yai, Songkhla 90110, Thailand. \\ ${ }^{3}$ Maharat Nakhon Ratchasima Hospital, Mueang, Nakhon Ratchasima 30000, Thailand. \\ Received 17 May 2019 • Revised 25 June 2019 • Accepted 27 June 2019 • Published online 16 August 2019
}

\section{Abstract:}

Objective: To estimate and compare the prevalences of anxiety at first and subsequent pregnancies and explore its associated factors.

Material and Methods: The study was conducted among multiparous women aged <34 years admitted to the postpartum ward in a regional hospital in northeastern Thailand during April-September 2014. Information on levels of anxiety, social support, financial stressors, self-esteem and socio-demographic characteristics was collected. Anxiety at the first pregnancy and the most recent pregnancy were assessed. Factors associated with the anxiety were analyzed by multiple logistic regression.

Results: Of the 447 women enrolled in the study, $24.0 \%$ had experienced high-level anxiety when becoming aware of being pregnant for the first pregnancy which was associated with the pregnancy being unplanned, high financial stressors and low social support. Sixty percent of the women felt that the degree of anxiety in their current pregnancy was lower. Decreased anxiety was more likely to be found in women who changed employment status from unemployed to employed, those with increased social support, and those with decreased financial stressors, and less likely in women with increased financial stressors.

Conclusion: One-fourth of the women reported anxiety at their first pregnancy which decreased in subsequent pregnancies. Social support and financial stressors during pregnancy should be explored and strategies on how to improve these conditions at antenatal care services should be studied.

Keywords: antenatal anxiety, social support, financial stressor, unplanned pregnancy

Contact: Prof. Tippawan Liabsuetrakul, M.D., Ph.D.

Epidemiology Unit, Faculty of Medicine, Prince of Songkla University,

Hat Yai, Songkhla 90110, Thailand.

E-mail: Itippawa@yahoo.com
J Health Sci Med Res 2019;37(4):259-268 doi: 10.31584/jhsmr.201959 www.jhsmr.org 


\section{Introduction}

During pregnancy and childbirth, women are faced with many physiological, hormonal and psychological changes. Failure to adapt to these changes increases the likelihood of psychological and emotional disturbances. Anxiety is one of the most common psychological problems during both the antenatal ${ }^{1,2}$ and postpartum periods ${ }^{3}$, and is a risk factor affecting health outcomes of both mothers and babies. ${ }^{4-6}$ The prevalence of antenatal anxiety has been reported variously ranging from 5.0 to $54.0 \%$, the variability being due to differences in gestational age, definitions of anxiety, sample groups or measurement scales and instruments. ${ }^{1,7-10}$ The prevalence of antenatal anxiety is high in developing countries where there are high prevalences of adolescent birth. ${ }^{11}$ Anxiety is most common during the first and third trimesters of pregnancy ${ }^{12}$ and a higher prevalence during pregnancy compared to the postpartum period has also been observed. ${ }^{13}$ In our study, antenatal anxiety was defined as the pregnancyspecific worries, concerns or fears about pregnancy and childbirth as well as the health of the infant and future parenting. ${ }^{12}$

Differences in the prevalences of anxiety between adolescents and adults have been shown. ${ }^{14-16}$ Antenatal anxiety is more likely to be found in women with anxiety and/or depressive disorders prior to pregnancy, those with low education, low self-esteem, low income, those who are unmarried and those with a greater number of previous abortions. ${ }^{8,17}$ A recent systematic review showed that women who had no partner or a lack of social support, those with an unplanned or unwanted pregnancy, those with one or more adverse life events, a high perceived stress level, or a past or present pregnancy complication were more likely to have antenatal anxiety and depression. ${ }^{13}$ Previous studies have found that a history of prior abortion or miscarriage in a previous pregnancy increased the risk of anxiety in subsequent pregnancies. ${ }^{18-20}$ However, this association was not found to be significant after adjusting for other associated factors. ${ }^{20}$ In addition, the study did not attempt to assess whether the anxiety would be decreased or increased if the women had passed the transition period of deciding whether to abort or to continue the pregnancy.

Another study found that women with untreated anxiety had a risk of comorbid depressive disorders with both immediate and long-term effects. ${ }^{21}$ The effect of maternal age on anxiety is still uncertain ${ }^{17,22,23}$, and there have been no studies investigating anxiety in the first and subsequent pregnancies in Thailand. Therefore, this study aimed to estimate the prevalence of anxiety in the first pregnancy and its changes from first to subsequent pregnancies and explore the factors associated with anxiety at first pregnancy and decreasing anxiety in subsequent pregnancies.

\section{Material and Methods}

\section{Study design and setting}

A historical cohort study was conducted from April to September 2014 at Maharat Nakhon Ratchasima Hospital, a regional hospital in the northeast of Thailand. This tertiary care hospital is located in Nakhon Ratchasima province which has a mixed economy including industry, agriculture, and retail outlets. This hospital serves a heterogeneous population of both poor and middleclass local and provincial residents with an average of 21,500 deliveries per year.

\section{Study participants}

Women aged 34 years or less who were admitted at the postpartum ward after delivery and had experienced at least one previous pregnancy within the past five years regardless of delivery status were included. Those who had had twins in the first pregnancy, who had a physical 
disability, neurological disorder, psychiatric disorder leading to barriers of communication, mental retardation or hearing loss were excluded. All women were screened by a nurse for eligibility for the study using a screening checklist.

To estimate the prevalence of anxiety in pregnancy, the one-group proportion formula was used based on an anxiety prevalence of $15.0 \%{ }^{10}$ and acceptable error of $5.0 \%$, which resulted in a minimum required sample size of 196 women. As we could find no available prevalences of decreased anxiety in subsequent pregnancy compared with the first pregnancy between adolescent and adult pregnancies, the prevalences of anxiety in adolescent and adult at their first pregnancies were considered for sample size calculation. The sample size was determined using the two-proportion formula for the prevalence of anxiety outcomes. Prevalences of anxiety for women having their first pregnancy as an adolescent pregnancy of $26.0 \%$ and for women having their first pregnancy as an adult of $11.0 \%$ were used. With a $95.0 \%$ confidence level, a power of $80.0 \%$, a ratio of adolescent to adult pregnancy of 2 to 3 and compensating for $10.0 \%$ incomplete data, at least 427 women (171 in the adolescent pregnancy group and 256 in the adult pregnancy group) were required.

\section{Variables and definitions}

The main outcome measure was anxiety status which was assessed for three time periods: T1 (the time when the woman became aware of being pregnant for the first pregnancy), T2 (the time when the woman made the decision to continue or terminate the pregnancy at the first pregnancy), and T3 (the time when the woman became aware of being pregnant at the most recent pregnancy).

The Postpartum Depression Risk Scale (PDRS) was chosen as the measurement tool in this study because this instrument examines anxiety, social support, financial stressors and self-esteem and has been validated among pregnant women for prediction of postpartum depression with a sensitivity of $80.0 \%$ and specificity of $74.0 \%{ }^{24}$ The PDRS has 8 items on anxiety, 10 items on social support, 3 items on financial stressors and 5 items on self-esteem. The anxiety in the PDRS refers to pregnancy-specific anxiety which measures the concerns or worries of pregnancy and future parenting and the perceptions of mental illness, sexual problems, frightening events and feeling stress. At $\mathrm{T} 1$, each anxiety item was rated with 5-rating scale as $1=$ “Never”, 2 ="1-2 times per month", 3 ="1 time per week", and $4=$ " 2 times per week" and 5="Almost every day. The anxiety scores in the study ranged from 8 to 40 of which a cut-off score of 16 was used to classify women as low or high anxiety. At T2 and T3, each item was measured in terms of the relative changes in anxiety, social support, financial stressors and self-esteem at T2 and T3 compared to T1 which were defined as increased, unchanged, or decreased. Independent variables measured in the first pregnancy included age group defined as either adolescent aged 10-19 years or adult aged 20-29 years, marital status, highest educational achievement, employment status, intention of pregnancy, outcome of pregnancy, social support, financial stressors, self-esteem, and living with the same partner of their first pregnancy; their partner's characteristics including age group, highest educational achievement, and employment status; and the respondent's mother's history of adolescent pregnancy and highest educational achievement. Intention of pregnancy was classified as either planning to become pregnant as planned pregnancy or unplanned pregnancy otherwise.

Factors assessed to be associated with a decrease in anxiety from $\mathrm{T} 1$ to $\mathrm{T} 3$ included change in age group from adolescent to adult between the first and current pregnancies, changes in employment status, outcome of first pregnancy, and changes in social support, financial stressors or self-esteem. Change in employment status was classified into four states: unemployed-unemployed, 
unemployed-employed, employed-employed, employedunemployed. Changes in social support, financial stressors and self-esteem were classified as increased, unchanged, or decreased.

\section{Data collection}

The enrollment criteria were checked against all delivered women until the required sample size number was met as calculated for both the adolescent and adult pregnancy groups at the first pregnancy. The eligible women were visited by the principal investigator during the first two or three postpartum days at the maternity ward who explained the study and invited them to participate in the study. Face-to-face interviews were conducted among the consenting women separately using a structured questionnaire in a private room.

\section{Data analysis}

Double data entry and validation were done using EpiData software. All analyses were performed using R Software version 3.1.2 (The R Foundation for Statistical Computing, Vienna, Austria). Prevalences and changes of anxiety were calculated as percentages. Factors associated with anxiety at first pregnancy and decreased anxiety were analyzed using a multiple logistic regression model which is presented in terms of adjusted odds ratios and $95 \%$ confidence intervals. $A$ p-value less than 0.05 was considered as significant.

\section{Ethical approval}

The study was approved by the Ethics Committee of the Faculty of Medicine, Prince of Songkla University (EC: 57-003-18-9) and the Maharat Nakhon Ratchasima Hospital Institutional Review Board. The details of the study were discussed with all eligible women and those who agreed to participate signed an informed consent form before data collection. For women aged less than 18 years, their guardian also signed the consent form.

\section{Results}

A total of 2,594 women who delivered from April to September 2014 were screened. A total of 527 women met the inclusion criteria, but four were excluded as two of them had had twins in the first pregnancy and the other two had a psychiatric disorder. Of the remaining 523 eligible women, 34 were discharged before they were approached and 42 declined to join the study. Finally, 447 women were interviewed (85.5\% response rate) of whom 180 were in the adolescent group and 267 in the adult group. The characteristics of the 447 women who participated in the study at their first pregnancy are shown in Table 1. Over half of the women were employed (59.7\%). Approximately half were unmarried and had achieved less than a high school level of education. More than half of the participants had an unplanned pregnancy $(62.0 \%)$. Approximately one-fourth of the women had terminated their first pregnancy. The majority of the women's partners were adult age and employed. Approximately one-fifth of the women became separated from their partner after they became pregnant.

Table 1 Characteristics of women and their partners and mothers at first pregnancy $(n=447)$

\begin{tabular}{lll}
\hline Characteristic & Number & Percent \\
\hline Women's characteristics & & \\
Age group & & \\
$\quad$ Adolescent (10-19 years) & 180 & 40.3 \\
$\quad$ Adult (20-29 years) & 267 & 59.7 \\
Marital status & & \\
$\quad$ Unmarried & 219 & 49.0 \\
$\quad$ Married & 228 & 51.0 \\
Highest educational achievement & & \\
$\quad$ Junior high school or lower & 217 & 48.5 \\
$\quad$ High school or higher & 230 & 51.5 \\
Employment status & & \\
$\quad$ Unemployed & 180 & 40.3 \\
$\quad$ Employed & 267 & 59.7 \\
Intention of pregnancy & & \\
$\quad$ Planned & 170 & 38.0 \\
$\quad$ Unplanned & 277 & 62.0 \\
\hline
\end{tabular}


Table 1 (continued)

\begin{tabular}{lll}
\hline Characteristic & Number & Percent \\
\hline Outcomes of pregnancy & & \\
$\quad$ Termination of pregnancy & 102 & 22.8 \\
$\quad$ Continuation of pregnancy to delivery & 345 & 77.2 \\
Living status with partner & & \\
$\quad$ Married and living together & 311 & 69.5 \\
$\quad$ Not married but still living together & 49 & 11.0 \\
$\quad$ Became separated from partner upon & 87 & 19.5 \\
$\quad$ becoming pregnant & & \\
Partner's characteristics & & \\
Age & & \\
$\quad$ Adolescent (10-19 years) & 90 & 20.1 \\
$\quad$ Adult (20-29 years) & 357 & 79.9 \\
Highest educational achievement & & \\
$\quad$ Junior high school or lower & 238 & 53.2 \\
$\quad$ High school or higher & 209 & 46.8 \\
Employment status & & \\
$\quad$ Unemployed & 33 & 7.4 \\
$\quad$ Employed & 414 & 92.6 \\
Women's mother's characteristics & & \\
History of adolescent pregnancy & & \\
$\quad$ No & 402 & 89.9 \\
$\quad$ Yes & 45 & 10.1 \\
Highest educational achievement & & \\
$\quad$ Primary school or lower & 380 & 85.0 \\
Junior high school or higher & 67 & 15.0 \\
\hline
\end{tabular}

Approximately a quarter of the women (23.5\%) had experienced a high level of anxiety during their first pregnancy. Women who had been adolescent when they became pregnant for the first time were significantly more likely to have experienced high anxiety compared to adults in univariate analysis $(28.9 \%$ vs $19.9 \%$, $p$-value $=$ 0.02) but the difference was not significant in multivariate analysis. Figure 1 presents the factors associated with high anxiety during the first pregnancy from the multiple logistic regression analysis. Unplanned pregnancy (adjusted odds ratio $(\mathrm{AOR}) 2.43,95 \%$ confidence interval $(\mathrm{Cl})$ 1.14-5.16), unemployed partner (AOR 4.09, 95\% Cl 1.5910.52) and stress (AOR 1.30, 95\% Cl 1.19-1.42) were associated with higher odds of anxiety. Women with social support had significantly lower odds of anxiety (AOR 0.84, 95\% Cl 0.78-0.90).

The changes of anxiety between the decision whether to terminate or continue the first pregnancy (T2) and when becoming aware of being pregnant at the most recent pregnancy (T3) compared to the anxiety felt when becoming aware of being pregnant for the first pregnancy

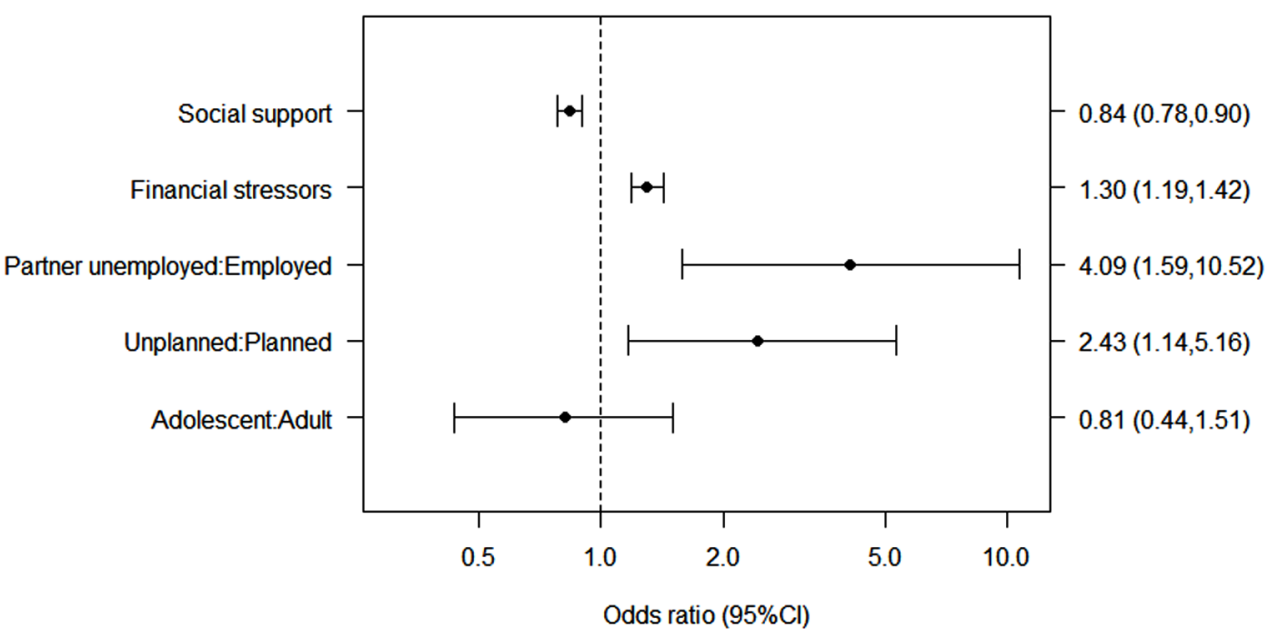

Figure 1 Odds ratios and 95\% confidence intervals of factors associated with anxiety at first pregnancy 
(T1) are shown in Figure 2. Slightly lower proportions of increased or unchanged anxiety were reported at T2 and T3 compared with T1, respectively. Two-thirds of the women had decreased anxiety at the most recent pregnancy compared to the first pregnancy.

The factors associated with a decrease in anxiety from the first pregnancy to the most recent pregnancy from the multiple logistic regression analysis are shown in
Figure 3. Women who changed employment status from unemployed to employed (AOR 2.60, 95\% Cl 1.15-5.91) and those with increased social support were more likely to have decreased anxiety (AOR 7.54, 95\% Cl 4.42-12.85). Women who had a decrease of financial stressors had higher odds of decreased anxiety (AOR 2.65, 95\% Cl 1.53-4.59), in contrast with those who had increased financial stressors (AOR 0.44, 95\% Cl 0.23-0.81).

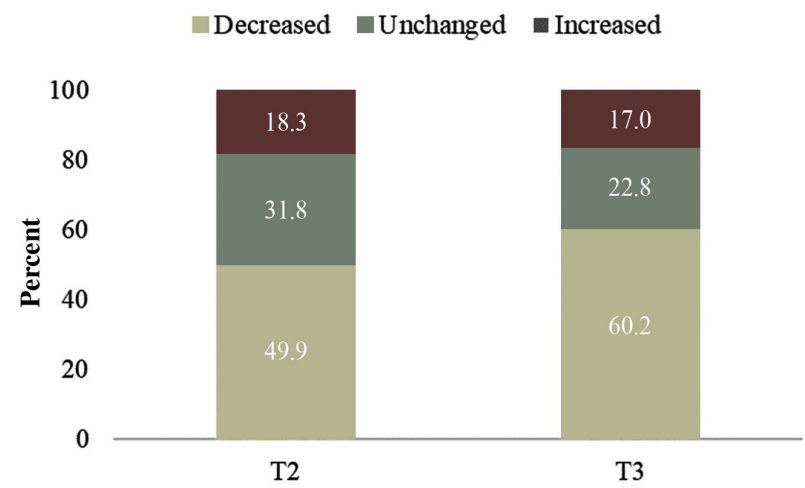

Figure 2 Proportion of women with anxiety when making the decision to continue or terminate the first pregnancy (T2) and becoming aware of being pregnant at the most recent pregnancy (T3) compared to when becoming aware of being pregnant at the first pregnancy $(\mathrm{T} 1)$

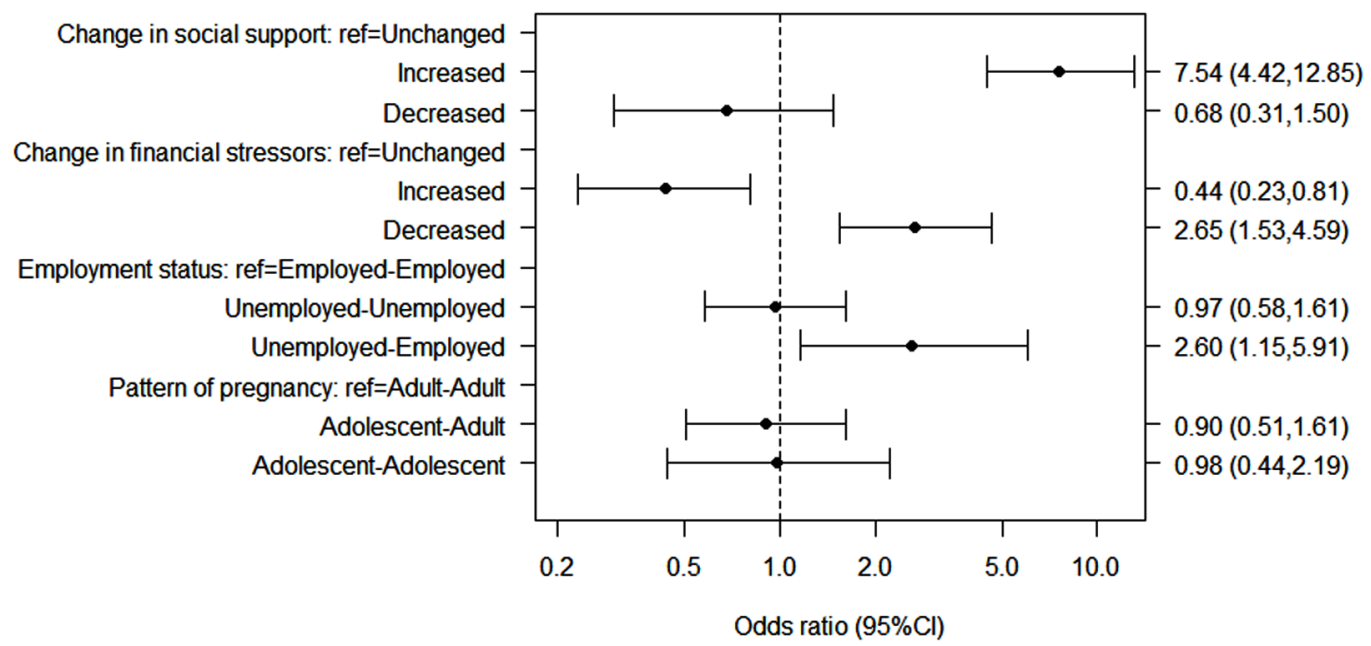

Figure 3 Odds ratios and 95\% confidence intervals of factors associated with decreased anxiety between the first pregnancy and the most recent pregnancy 


\section{Discussion}

A high prevalence of antenatal anxiety among women during their first pregnancy was found. Factors associated with anxiety were unplanned pregnancy, high financial stress and low social support. Women who became employed, had decreased financial stressors, and increased social support had decreased anxiety levels in their subsequent pregnancies. Adolescent women had a higher level of anxiety compared to adults but the difference was not significant after adjusting for unplanned pregnancy and low socioeconomic support.

The prevalence of anxiety among women during their first pregnancy in our study was twice that reported by a study conducted in Malaysia ${ }^{7}$, similar to that reported by a prospective study in Italy ${ }^{9}$ and lower than in studies conducted in Brazil and Hong Kong. ${ }^{1,8}$ This could be explained by the different methods used to measure anxiety in each study. The studies in Malaysia and Italy used a diagnostic clinical interview, while the studies from Brazil and Hong Kong used a self-reported screening tool. Apart from the diagnostic criteria and tools used, differences in the timing of assessment (first, second or third trimester) and the study populations may also have influenced the results. One study reported a high prevalence of anxiety in pregnant women who were young, those living in low-middle income countries or who had a history of drinking. ${ }^{25}$

Adolescent women reported a higher prevalence of antenatal anxiety compared to adults; a result similar to a previous study in Thailand. ${ }^{10}$ Likewise, studies in Sweden and Canada found that the risk of antenatal anxiety in women aged under 25 years was two times higher compared to older aged women. ${ }^{25,26}$ However, in our study, the difference was not significant after adjusting for other factors. Social support and financial stressors were strongly associated factors for antenatal anxiety in our study, which was consistent with the findings of a systematic review ${ }^{13}$ and a prospective study conducted in Germany. ${ }^{17}$

Unplanned pregnancy was a significant risk factor for antenatal anxiety during the first pregnancy, consistent with a recent systematic review. ${ }^{13}$ A study in Canada found that unplanned pregnancy was a significant predictor for depression, but not anxiety. ${ }^{26}$ Unplanned pregnancy is a concern because it can lead not only to mental health problems but also adverse pregnancy outcomes for a woman and her newborn. ${ }^{27,28}$ A partner's unemployed status was also found to be a significant factor, a finding similar to a previous study ${ }^{29}$ which could be explained by understanding that a partner's income from employment is the main part of the income for most families, and lack of income when a partner is unemployed may affect financial stressors and a woman's anxiety.

The occurrence of anxiety when a woman recognizes her first pregnancy is a normal psychological phenomenon $^{30}$, but this condition can become a pathological condition if the adaptation to the new circumstance is limited and new pressures of becoming pregnant with insufficient support are overwhelming. ${ }^{18}$ The decreasing levels of anxiety in subsequent pregnancies in our study can be explained by the women's experiences of pregnancy and predictive conditions during pregnancy. ${ }^{29}$ In contrast, negative experiences of a previous pregnancy could lead to the risk of anxiety. ${ }^{31,32}$

This study found that women with a high risk of anxiety at the first pregnancy had increasing anxiety regardless of their decision to continue or terminate the pregnancy. This finding suggests that anxiety levels should be measured at the first antenatal care visit with close observation and more support given to women at high risk of anxiety. We found that increased social support, decreased financial stressors and changed employment status during the most recent pregnancy compared to the first pregnancy could decrease the level of anxiety. 
There are no previous studies on changes in exposures and anxiety levels in the same women at first and subsequent pregnancies and their associated factors from Thailand. We conducted a historical cohort study, rather than a prospective study, to be able to include women who had had at least two pregnancies so there would be no need for waiting for a subsequent pregnancy, which led to some limitations in the study. First, the first pregnancy occurred as far back as 5 years, thus memories of anxiety, social support, financial stressors and selfesteem during the first pregnancy may have led to recall bias. However, we pretested the questionnaire before the study was conducted and the women all confirmed that they could remember their first pregnancy accurately. Second, this study was facility-based and only women who delivered in their most recent pregnancy in this hospital were included, and thus the results cannot be generalized across other populations in Thailand or in other countries. Third, the gestational age at recognition of the woman's first pregnancy was not obtained so the effect of gestational age on the occurrence of anxiety could not be determined. Fourth, some eligible women were not included in the study due to their short hospital admission after delivery and limited time for an interview, however, there were only a few of these $(<15.0 \%)$ and there were no significant differences in their characteristics compared with those who participated in the study. Finally, domestic violence or abuse before or during pregnancy, which would certainly be a source of anxiety if present in the woman's life, were not included in the survey, due to the sensitivity of this kind of situation, and the difficulty of obtaining reliable information.

\section{Conclusion}

The prevalence of antenatal anxiety was high in the first and subsequent pregnancies in both adolescent and adult pregnant women. Women who change their employment status from unemployed to employed and who have decreased financial stressors and increased social support were found to have decreased anxiety levels in their subsequent pregnancies. Women with unplanned pregnancies and who feel social pressure during their pregnancies need careful attention, not only by doctors and nurses providing health services to improve the mental health of the women, but also to encourage the women's families to provide adequate support. Future prospective research exploring other potential risk factors of anxiety in pregnancy such as physical health status, influence of health services or social support from the community, should be undertaken.

\section{Acknowledgement}

This work is a part of the first author's doctoral thesis in Epidemiology at Prince of Songkla University. The authors wish to thank the study participants for their contribution to the research and all staff at the postpartum ward, delivery room and breastfeeding clinic of Maharat Nakhon Ratchasima Hospital, Nakhon Ratchasima, Thailand.

\section{Funding sources}

The study was funded by the Health Intervention and Technology Assessment Program and the Postgraduate Fund of the Graduate School, Prince of Songkla University, Thailand.

\section{Conflict of interest}

All authors declare no conflicts of interest.

\section{References}

1. Lee AM, Lam SK, Sze Mun Lau SM, Chong CS, Chui HW, Fong DY. Prevalence, course, and risk factors for antenatal anxiety and depression. Obstet Gynecol 2007;110:1102-12.

2. Teixeira C, Figueiredo B, Conde A, Pacheco A, Costa R. 
Anxiety and depression during pregnancy in women and men. J Affect Disord 2009;119:142-8.

3. Ross LE, McLean LM. Anxiety disorders during pregnancy and the postpartum period: a systematic review. J Clin Psychiatry 2006;67:1285-98.

4. Ding $X X$, Wu YL, Xu SJ, Zhu RP, Jia XM, Zhang SF, et al. Maternal anxiety during pregnancy and adverse birth outcomes: a systematic review and meta-analysis of prospective cohort studies. J Affect Disord 2014;159:103-10.

5. Kurki T, Hiilesmaa V, Raitasalo R, Mattila H, Ylikorkala O. Depression and anxiety in early pregnancy and risk for preeclampsia. Obstet Gynecol 2000;95:487-90.

6. Martini J, Knappe S, Beesdo-Baum K, Lieb R, Wittchen HU. Anxiety disorders before birth and self-perceived distress during pregnancy: associations with maternal depression and obstetric, neonatal and early childhood outcomes. Early Hum Dev 2010;86:305-10.

7. Fadzil A, Balakrishnan K, Razali R, Sidi H, Malapan T, Japaraj RP, et al. Risk factors for depression and anxiety among pregnant women in Hospital Tuanku Bainun, Ipoh, Malaysia. Asia Pac Psychiatry 2013;5(Suppl 1):S7-13.

8. Faisal-Cury A, Rossi Menezes P. Prevalence of anxiety and depression during pregnancy in a private setting sample. Arch Womens Ment Health 2007;10:25-32.

9. Giardinelli L, Innocenti A, Benni L, Stefanini MC, Lino G, Lunardi $\mathrm{C}$, et al. Depression and anxiety in perinatal period: prevalence and risk factors in an Italian sample. Arch Womens Ment Health 2012;15:21-30.

10. Piyasil V. Anxiety and depression in teenage mothers: a comparative study. J Med Assoc Thai 1998;81:125-9.

11. United Nations. The millennium development goals report 2012. New York: UN; 2012.

12. Madhavanprabhakaran GK, D’Souza MS, Nairy KS. Prevalence of pregnancy anxiety and associated factors. Int $\mathrm{J}$ Africa Nurs Sci 2015;3:1-7.

13. Biaggi A, Conroy S, Pawlby S, Pariante CM. Identifying the women at risk of antenatal anxiety and depression: a systematic review. J Affect Disord 2016;191:62-77.

14. Bodecs T, Szilagyi E, Cholnoky P, Sandor J, Gonda X, Rihmer $Z$, et al. Prevalence and psychosocial background of anxiety and depression emerging during the first trimester of pregnancy: data from a Hungarian population-based sample. Psychiatr Danub 2013;25:352-8.
15. Faisal-Cury A, Menezes $\mathrm{P}$, Araya R, Zugaib M. Common mental disorders during pregnancy: prevalence and associated factors among low-income women in Sao Paulo, Brazil: depression and anxiety during pregnancy. Arch Womens Ment Health 2009;12:335-43.

16. Nasreen HE, Kabir ZN, Forsell Y, Edhborg M. Prevalence and associated factors of depressive and anxiety symptoms during pregnancy: a population-based study in rural Bangladesh. BMC Womens Health 2011;11:22.

17. Martini J, Petzoldt J, Einsle F, Beesdo-Baum K, Hofler M, Wittchen HU. Risk factors and course patterns of anxiety and depressive disorders during pregnancy and after delivery: a prospective longitudinal study. J Affect Disord 2015;175: 385-95.

18. Woods-Giscombe CL, Lobel M, Crandell JL. The impact of miscarriage and parity on patterns of maternal distress in pregnancy. Res Nurs Health 2010;33:316-28.

19. Fertl KI, Bergner A, Beyer R, Klapp BF, Rauchfuss M. Levels and effects of different forms of anxiety during pregnancy after a prior miscarriage. Eur J Obstet Gynecol Reprod Biol 2009; 142:23-9.

20. Bicking Kinsey C, Baptiste-Roberts K, Zhu J, Kjerulff KH. Effect of previous miscarriage on the maternal birth experience in the First Baby Study. J Obstet Gynecol Neonatal Nurs 2013; 42:442-50.

21. van Beljouw IM, Verhaak PF, Cuijpers P, van Marwijk HW, Penninx BW. The course of untreated anxiety and depression, and determinants of poor one-year outcome: a one-year cohort study. BMC Psychiatry 2010;10:86.

22. Figueiredo B, Pacheco A, Costa R. Depression during pregnancy and the postpartum period in adolescent and adult Portuguese mothers. Arch Womens Ment Health 2007; 10:103-9.

23. Giardino J, Gonzalez A, Steiner M, Fleming AS. Effects of motherhood on physiological and subjective responses to infant cries in teenage mothers: a comparison with nonmothers and adult mothers. Horm Behav 2008;53:149-58.

24. Liabsuetrakul T, Vittayanont A, Pitanupong J. Clinical applications of anxiety, social support, stressors, and self-esteem measured during pregnancy and postpartum for screening postpartum depression in Thai women. J Obstet Gynaecol Res 2007;33:333-40.

25. Rubertsson C, Hellstrom J, Cross M, Sydsjo G. Anxiety in early 
pregnancy: prevalence and contributing factors. Arch Womens Ment Health 2014;17:221-8.

26. Bayrampour H, McDonald S, Tough S. Risk factors of transient and persistent anxiety during pregnancy. Midwifery 2015;31:582-9.

27. Singh A, Singh A, Mahapatra B. The consequences of unintended pregnancy for maternal and child health in rural India: evidence from prospective data. Matern Child Health J 2013; 17:493-500.

28. Hayatbakhsh MR, Najman JM, Khatun M, Al Mamun A, Bor W, Clavarino A. A longitudinal study of child mental health and problem behaviours at 14 years of age following unplanned pregnancy. Psychiatry Res 2011;185:200-4.

29. Karmaliani R, Asad N, Bann CM, Moss N, McClure EM, Pasha $\mathrm{O}$, et al. Prevalence of anxiety, depression and associated factors among pregnant women of Hyderabad, Pakistan. Int $\mathrm{J}$ Soc Psychiatry 2009;55:414-24.

30. Condon JT, Boyce P, Corkindale CJ. The first-time fathers study: a prospective study of the mental health and wellbeing of men during the transition to parenthood. Aust N Z J Psychiatry 2004;38:56-64.

31. Dipietro JA, Costigan KA, Sipsma HL. Continuity in self-report measures of maternal anxiety, stress, and depressive symptoms from pregnancy through two years postpartum. J Psychosom Obstet Gynaecol 2008;29:115-24.

32. Gong X, Hao J, Tao F, Zhang J, Wang H, Xu R. Pregnancy loss and anxiety and depression during subsequent pregnancies: data from the C-ABC study. Eur J Obstet Gynecol Reprod Biol 2013;166:30-6. 